\title{
Influence of microtexture on ultrasonic reflection in Ti-6Al-4V alloy hot-forged in $\alpha+\beta$ region
}

\author{
Yoshinori Ito ${ }^{1,4,},{ }^{*}$ Hiroyuki Takamatsu ${ }^{2}$, Shogo Saeki ${ }^{3}$, Nobuhiro Tsuji \\ ${ }^{1}$ Materials Research Laboratory, Kobe Steel, Ltd., Japan, ${ }^{2}$ Production Systems Research Laboratory, Kobe Steel, Ltd., Japan, \\ ${ }^{3}$ Titanium Division, Kobe steel, Ltd., Japan, ${ }^{4}$ Department of Materials Science and Engineering, Kyoto University, Japan, \\ *Corresponding author, e-mail address: ito.yoshinori@kobelco.com
}

\section{$\underline{\text { Abstract }}$}

Morphological change in regions with microtexture, i.e., macrozones, in Ti-6Al-4V alloy after hot-forging in $\alpha+\beta$ phase region was analyzed using SEM/EBSD technique, and then the correlation with ultrasonic reflection was investigated. Starting material had colony-type microstructure consisting of lamellar- $\alpha$ with the same crystallographic orientation. Forging (cogging and upsetting) was conducted in $\alpha+\beta$ phase region to produce a cylindrical material with smaller cross section, followed by air cooling. Thus, resulting microstructure consisted of granular- $\alpha$. To estimate local change in elastic modulus within the materials, we analyzed crystallographic orientations of $\alpha$-phase using SEM/EBSD in terms of an inclination angle of c-axis. The regions having the nearly common crystallographic orientations of $\alpha$-phase were found. Shapes of such regions with microtexture were elongated along the axial direction, which formed quasi-periodic variation of the inclination angle, i.e., elastic modulus, of $\alpha$-phase along the radial direction of the forged material. Ultrasonic measurement was conducted at a frequency of $5 \mathrm{MHz}$ along the radial direction and the axial direction. Relationship between the morphology of regions having microtexture and the amplitude of backscattered noises was evaluated. It was considered that the quasi-periodicity of the inclination angle correlated with the amplitude of backscattered noises in ultrasonic measurement.

\section{Introduction}

Titanium alloys are used in aerospace industry due to its light-weight and high-strength properties. Among them, $\alpha+\beta$ type titanium alloys are used critical parts, such as compressor discs, in aero engines. Failures of the critical parts result in serious accidents, so that ultrasonic inspection is conducted to ensure higher reliability. It is known that forging products of titanium alloys have difficulty in the inspection because backscattered noises is generated due to its microstructural inhomogeneity, and thus incident signal caused by defects might be masked by the noises [1]-[3].

In the last two decades, regions having microtexture of $\alpha$-phase in titanium alloy products, which is referred to as "macrozones", have been focused on [4]-[8]. In the regions having microtexture, crystallographic orientations of $\alpha$-phase are similar. Therefore, the regions act as one large grain, even though each grains and platelets of $\alpha$ phase is as small as $10 \mu \mathrm{m}$ or so. As results of several prior studies, presence of the microtexture is considered to degrade fatigue properties [4], [5] and also to cause backscattered noises in ultrasonic inspection [9]-[11].

In spite of prior studies, relationship between the regions having microtexture and amplitude of backscattered noises is still unclear, because of limited experimental works. Present authors have investigated characterization of microtexture and its influence on ultrasonic reflection in titanium alloys. We studied the backscattered noise in Ti-6Al-2Sn-4Zr-6Mo (Ti-6246) alloy upset-forged in $\beta$-single-phase region, thus its microstructure consisted of platelets of $\alpha$-phase [12]. In our previous study, ultrasonic measurement conducted both parallel and perpendicular to the forging axis directions. Along the forging axis direction, amplitude of backscattered noises strongly depended on the forging reduction in height, and the noises increased as the reduction increased up to $67 \%$. On the other hand, in perpendicular to the forging axis direction, the amplitude of backscattered noises kept low regardless of the forging reduction. Therefore, anisotropy of the noise became larger with increasing the forging reductions [12]. Recently, we have investigated evolution of microtexture of $\alpha$-phase in the Ti-6246 alloy hot-forged in $\beta$-single-phase region, and then discussed relationship of size of the regions having microtexture and the amplitude of backscattered noises. Detail results will be reported elsewhere. We have also investigated microtexture in Ti-6Al-4V alloy hot-forged in $\alpha+\beta$ phase region, thus consisting of granular- $\alpha$. In this study, we conducted ultrasonic measurement in the forging products of Ti- $6 \mathrm{Al}-4 \mathrm{~V}$ alloy, and then correlation between microtexture and the amplitude of backscattered noises was discussed.

\section{$\underline{\text { 2. Experimental procedures }}$}

Material used in this study was Ti-6Al-4V alloy billet $405 \mathrm{~mm}$ in diameter. Starting material had colony-type microstructure consisting of lamellar- $\alpha$ which was obtained by heat treatment in $\beta$-single-phase region followed by air cooling to room temperature. In a series of our investigation regarding Ti-6Al-4V alloy, two types of forging process such as cogging and upsetting were used. The forging with four different magnitude of deformation was conducted in $\alpha+\beta$ phase region to produce cylindrical materials with smaller cross sections. In this study, one of the forged materials was selected for investigation. The starting material and the forged material were referred to as Sample-A and Sample-B, respectively, hereafter. Rectangular specimens were cut from the cylindrical materials for microstructural observation and ultrasonic measurement. Schematic illustration in Fig. 1 shows a position where the specimens were cut. Ultrasonic measurement was conducted at a frequency of $5 \mathrm{MHz}$ in two orthogonal directions of the rectangle specimens. First direction corresponded to the radial direction of the cylindrical material $\left(\mathrm{R}_{1}\right)$, and second direction was parallel to the axial direction of the cylindrical material (A.D.). Specimens and a transducer were immersed in water, and then the transducer sent incident wave normal to a surface of the specimen. Ultrasonic waves reflected backward, i.e., backscattered signal, were received by the transducer. Backscattered signals were amplified by gain value of $40 \mathrm{~dB}$. SEM/EBSD analysis was conducted on a section perpendicular to $\mathrm{R}_{1}$.

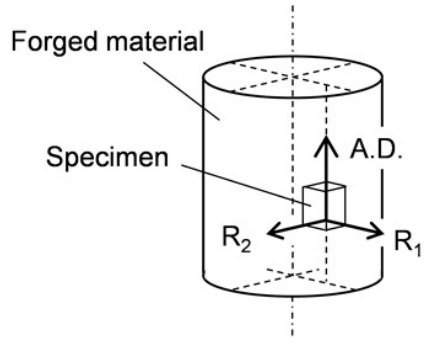

A.D. : Axis direction

$\mathrm{R}_{1}, \mathrm{R}_{2}:$ Radial direction

Fig. 1 Schematic illustration showing a position where specimens for ultrasonic measurement and SEM/EBSD analysis were taken from cylindrical materials of Ti6Al-4V alloy.

(C) The Authors, published by EDP Sciences. This is an open access article distributed under the terms of the Creative Commons Attribution License 4.0 (http://creativecommons.org/licenses/by/4.0/). 


\section{Results and discussions}

(1) Optical microstructures

Optical microstructures of Sample-A and Sample-B are shown in Fig. 2. Sample-A consists of lamellar- $\alpha$ that thickness is a few $\mu \mathrm{m}$ (Fig. 2 (a)), whereas Sample-B comprises of granular- $\alpha$ that size is as large as approximately $20 \mu \mathrm{m}$ (Fig. 2 (b)).

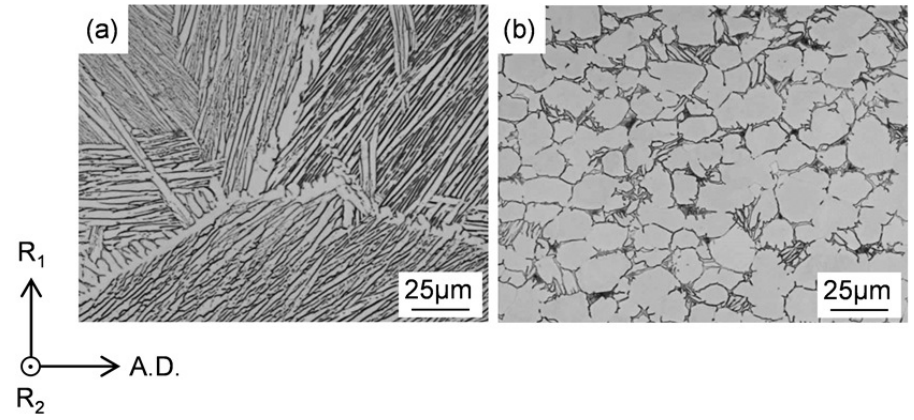

Fig. 2 Optical microstructures of Ti-6Al-4V. (a) Sample-A: After heat treatment in $\beta$-single-phase-region (without forging). (b) Sample-B: Forged in $\alpha+\beta$ phase region after the heat treatment in $\beta$-single-phaseregion.

(2) Backscattered noise

Backscattered signals of Sample-A and Sample-B were obtained in two orthogonal directions. As generally known, ultrasonic waves are reflected at boundaries between two domains having different elastic modulus and/or density. Thus, reflections occur at both a front surface and a bottom surface of specimens, which are referred to as FR and BR, respectively, hereafter. Microstructural inhomogeneity in materials also results in reflection. Backscattered signals caused by the microstructural inhomogeneity are observed between FR and BR, which can be recognized as "backscattered noises". In this study, root mean squared amplitude of backscattered signals was acquired. And then, averaged values of the amplitude of backscattered noises were obtained within a center area of the specimen. Results are summarized in Fig. 3. Blue bars show the amplitude of average noises along $R_{1}$ and red bars indicate that parallel to A.D. As for Sample-A, average noises measured in both directions shows similar magnitude of values, which indicates the backscattered noises of Sample-A is isotropic with respect to measured directions. On the other hand, the amplitude of average noises in Sample-B exhibit anisotropic features. It indicates that the amplitude of average noises in $\mathrm{R}_{1}$ was increased, whereas that in the A.D. was decreased as a result of the forging

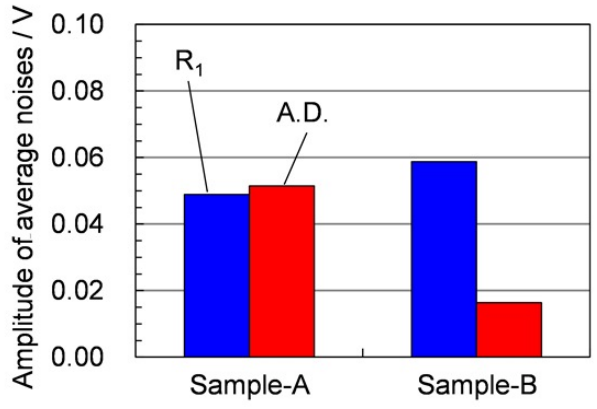

Fig. 3 Amplitude of average noises of Sample-A and Sample-B. Ultrasonic measurement was conducted along two orthogonal directions. $R_{1}$ : parallel to the radial direction of cylindrical materials, A.D.: parallel to the axial direction of cylindrical materials.

To clarify reasons for evolution of the anisotropic feature in Sample-B, this section will focus on microtexture development, and then its effects on generation of the backscattered noises will be discussed. Ti-6Al-4V alloy consists of approximately $95 \%$ of $\alpha$-phase and residual $\beta$-phase. Thus, we will consider crystallographic orientation of $\alpha$-phase. As generally known, $\alpha$-phase has hep lattice and its elastic modulus depends on an inclination angle of c-axis from a certain direction with concern, i.e., a reference direction. Fisher et al. reported elastic constants of $\alpha$-phase in $\mathrm{Ti}$ [13]. Based on the Fisher's results, change in elastic modulus of $\alpha$-phase with respect to an inclination angle was obtained by rotation matrix (Fig.4). In supplemental schematic drawings in Fig. 4, a reference direction is the vertical direction. Elastic modulus shows the maximum value at the inclination angle of zero, i.e., c-axis is parallel to the vertical direction, and then decreases with increasing the inclination angle. At the angle of c-axis tilting perpendicular to the vertical direction, elastic modulus reaches the minimum value. Accordingly, the change in the inclination angle results in variation of elastic modulus. Therefore, boundaries between two regions having different microtexture are expected to cause reflection of ultrasonic wave. SEM/EBSD analysis was conducted to focus on crystallographic orientation of $\alpha$-phase, especially in the inclination angle. 


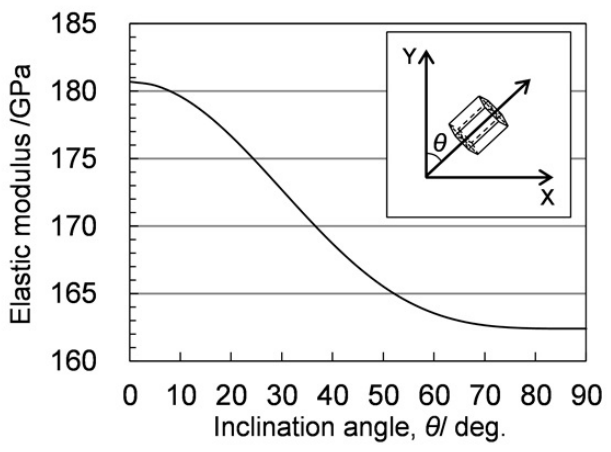

Fig. 4 Change in elastic modulus of $\alpha$-phase with respect to the inclination angle between $\mathrm{c}$-axis and a reference direction, i.e., the vertical direction in this figure.

IPF maps and corresponding pole figures of Sample-A and Sample-B are summarized in Fig. 5 and Fig. 6, respectively. The color in the IPF maps indicates the crystallographic orientation parallel to $\mathrm{R}_{1}$ at each measured point, according to the key stereographic triangles shown in figures. As indicated in Fig. 2 (a), Sample-A has lamellar structure of $\alpha$-phase. Fig. 5 (a) shows large colony structure in which adjacent lamellar- $\alpha$ have the same crystallographic orientation. Shape of colonies is blocky and its size is approximately $2000 \mu \mathrm{m}$. Orientation of each colony is relatively random with each other. Fig. 5 (b-d) show (0001) pole figures of area-1 through area-3 indicated by white squares in the IPF map. Shape of the areas is rectangle elongating perpendicular to $R_{1}$. It can be seen that each area contains parts of several colonies. Pole figures of extracted areas confirm that Sample-A doesn't have specific texture, but there are several isolated spots of c-axes having different crystallographic orientations, i.e., several inclination angle from $\mathrm{R}_{1}$. Area-4 which is a square area elongating perpendicular to A.D. was also analyzed (Fig. 5 (a), (e)). Corresponding pole figure exhibits that there are also several isolated spots of c-axes, i.e., several inclination angle from A.D., in the area. It suggests that the amplitude of backscattered noises generating along $\mathrm{R}_{1}$ and A.D. are comparable, which is consistent with the experimental result in Fig. 3. Fig. 6 shows IPF map and corresponding pole figures in Sample-B. As shown in Fig. 2 (b), Sample-B consists of granular- $\alpha$. Fig. 6 (a) indicates that there are regions in which adjacent $\alpha$-grains have similar crystallographic orientation, i.e., microtexture. Shape of regions having microtexture is elongated perpendicular to $R_{1}$. Fig. 6 (b-f) show (0001) pole figures of area-5 through area-9 indicated by white squares in the IPF map. As shown in Fig. 6 (a) and Fig. 6 (b), area-5 consists of almost one region having microtexture colored in red, because of elongated morphology of the regions. Thus, pole figure shows strong texture whose c-axes align to a direction between $\mathrm{R}_{1}$ and A.D. (Fig. 6 (b)). In area-8, c-axes align almost perpendicular to $\mathrm{R}_{1}$, which exhibits relatively sharp intensity as well (Fig. 6 (e)). Area-9 also have texture whose c-axes parallel to $\mathrm{R}_{1}$, which indicates that the region colored in red is the main component in the area (Fig. 6 (f)). Intensity of texture in area- 6 and area-7 are relatively weak, because several regions having different microtexture exist in the extracted areas (Fig. 6 (c), (d)). But the highest intensity of c-axes can be recognized at an angle perpendicular to $\mathrm{R}_{1}$ in area- 6 (Fig. 6 (c)), and at an angle tilting by about $50^{\circ}$ to $\mathrm{R}_{1}$ from the normal direction of pole figure, i.e., $R_{2}$ (Fig. 6 (d)). Accordingly, the development of microtexture leads to formation of band structure which consists of layers elongated to A.D. Adjacent layers have microtexture with different inclination angle. Therefore, it is expected that the band structure generates quasi-periodic change in elastic modulus along $\mathrm{R}_{1}$. Thus, when ultrasonic wave propagates parallel to $R_{1}$, backscattered reflection is expected to occur so as to generate backscattered noises. Fig. 6 (g) indicates ( 0001$)$ pole figure of area-10 indicated by a white square which elongate perpendicular to A.D., and shows that relatively weak texture form in the area. According to this pole figure and the IPF map, little change in elastic modulus is expected along A.D. So, when ultrasonic wave propagates along A.D., significant backscattered reflection isn't expected to happen. The above expectations are coincide with the experimental results in Fig. 3 which showed higher average noise along $\mathrm{R}_{1}$ rather than along A.D. in Sample-B.

Consequently, it is considered that the formation of band structure which consists of elongated regions having microtexture attributes to generation of the anisotropy of the average noises. The amplitude of backscattered noises is expected to depend on both thickness of the region having microtexture and magnitude of quasi-periodic change in elastic modulus. To clarify detail effects, more precise characterization of microtexture will be investigated.

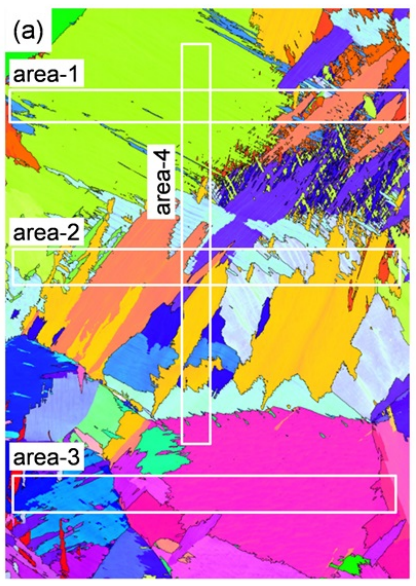

$2000 \mu \mathrm{m}$
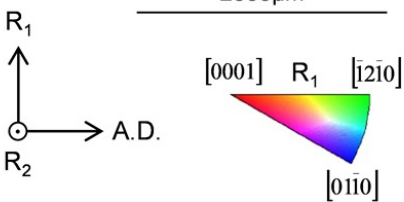

(b)

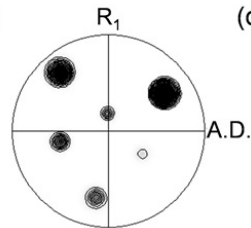

(d)

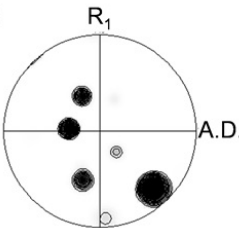

(c)
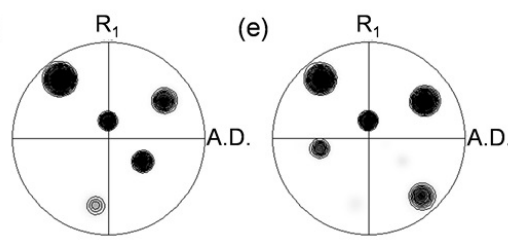

$\mathrm{R}_{1}, \mathrm{R}_{2}$ : Radial direction

A.D. : Axial direction

Fig. 5 (a) IPF map and (b-e) corresponding (0001) pole figures of Sample-A. Pole figures are obtained in extracted areas indicated by white squares in the IPF map. Each corresponding area is (b) area-1, (c) area-2, (d) area-3 and (e) area-4, respectively. 


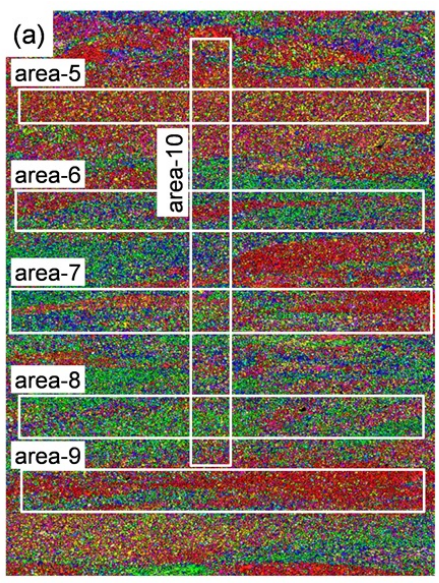

$2000 \mu \mathrm{m}$

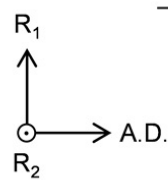

$\left.[0001] \quad R_{1} \mid 1210\right]$

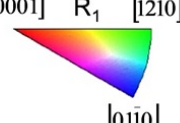

(b)

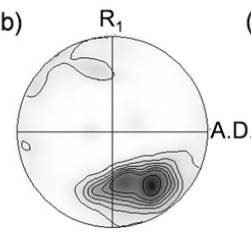

(c)
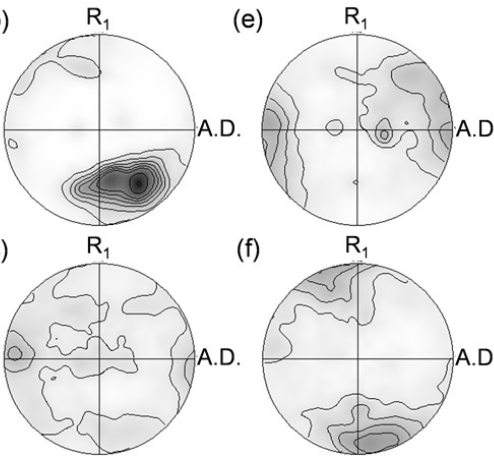

$\mathrm{R}_{1}$

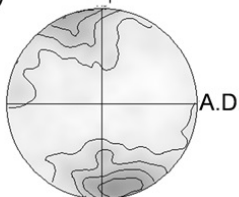

(d)
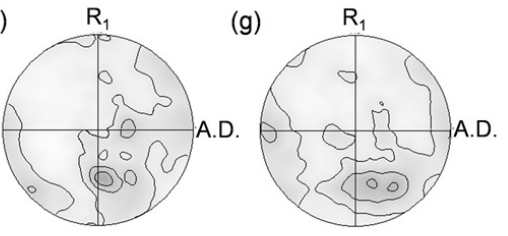

$\mathrm{R}_{1}, \mathrm{R}_{2}$ : Radial direction

A.D. : Axial direction

Fig. 6 (a) IPF map and (b-g) corresponding (0001) pole figures of Sample-B. Pole figures are obtained in extracted areas indicated by white squares in the IPF map. Each corresponding area is (b) area-5, (c) area-6, (d) area-7, (e) area-8, (f) area-9 and (g) area-10, respectively.

\section{Conclusions} follows.

Microtexture in Ti-6Al-4V alloy hot-forged in $\alpha+\beta$ region was investigated and then its influence on ultrasonic reflection was discussed. The main results are as

(1) Ti-6Al-4V alloy heat treated in $\beta$-single-phase-region, i.e., Sample-A, showed isotropic amplitude of average noises with respect to the directions of ultrasonic measurement. On the other hand, Ti-6Al-4V alloy hot-forged in $\alpha+\beta$ region after the heat treatment in $\beta$-single-phase-region, i.e., Sample-B, had anisotropic feature of average noises, that is, the forging led to increasing the average noises in $R_{1}$, whereas decreasing the average noises in A.D.

(2) Sample-A consisted of blocky colonies whose size was approximately $2000 \mu \mathrm{m}$. Pole figures of the extracted areas in IPF map indicated that each area contained parts of several colonies having different inclination angles of c-axes. Sample-B comprised of several regions having microtexture elongated parallel to A.D. Pole figures of the extracted areas in IPF map exhibited that most of areas had texture, and adjacent areas had different inclination angles of c-axes with each other along $\mathrm{R}_{1}$.

(3) The development of microtexture led to the formation of band structure elongated to A.D. in Sample-B. The band structure caused quasi-periodic change in elastic modulus along $R_{1}$, while significant change in elastic modulus wasn't expected along A.D. It was expected that the formation of the band structure attributed to the anisotropic feature of average noises in Sample-B, in spite of isotropic average noises in Sample-A.

\section{References}

[1] F. J. Margetan, Review of Progress in Quantitative Nondestructive Evaluation, 1992, vol.11B, pp.1717-1724.

[2] F. J, Margetan, J Nondestructive Evaluation, 1994, vol.13, pp.111.

[3] Blodgett, J. Nondestruct Eval. 2001, vol. 20, pp.1.

[4] A.P. Woodfield, M.D. Gorman, R.R. Corderman, J.A. Sutliff, and B. Yamrom: Titanium 95 Science and Technology, 1995, pp.1116-1123.

[5] K. Le Biavant, S. Pommier, and C. Prioul: Fatigue Fract. Eng. Mater. Struct., 2002, vol. 25, pp. 527-545.

[6] L. Germain, N. Gey, M. Humbert, P. Bocher, and M. Jahazi: Acta Mater., 2005, vol. 53, pp. 3535-3543.

[7] L. Germain, N. Gey, M. humbert, P. Vo, M. Jahazi, and P. Bocher: Acta Mater., 2008, vol. 56, pp. 4298-4308.

[8] N. Gey, P. Bocher, E. Uta, L. Germain, and M. Humbert: Acta Mater., 2012, vol. 60, pp. 2647-2655.

[9] P. D. Panetta, R.B. Thompson, and F.J. Margetan, Review of Progress in Quantitative Nondestructive Evaluation, 1998, vol. 17, pp. 89-96.

[10] M.F.X. Gigliotti, B.P. Bewlay, J.B. Deaton, R.S. Gilmore, and G.A. Salishcev, Metall. Mater. Trans. A, 2000, vol. 31A, pp. $2119-2125$.

[11] M. Humbert, A. Moreau, E. Uta, N. Gey, P. Bocher, and C. Bescond: Acta Mater., 2009, vol. 57, pp. 708-714.

[12] Y. Ito, H. Takamatsu, and K. Kinoshita: Proceedings of the 13th World Conference on Titanium, 2016, pp. 885-888.

[13] E.S. Fisher, and C.J. Renken, Physical Review, 1964, vol. 135, pp. 482-494 